\title{
S-Equol ameliorates insulin secretion failure through Chrebp/Txnip signaling via modulating PKA/PP2A activities
}

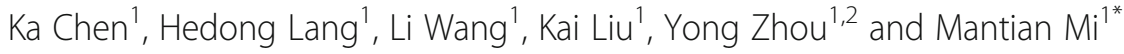

\begin{abstract}
Background: S-Equol, produced from daidzein by gut microbiota, has been suggested as an potential anti-diabetic agent, but the underlying mechanisms remain unclear. Recent evidences demonstrated that carbohydrate response element-binding protein (Chrebp)/Thioredoxin-interacting protein (Txnip) signaling played central roles on diabetes progression, particularly in relation to the function maintenance and apoptosis of pancreatic $\beta$-cell. Here, we investigated the effects of S-Equol on $\beta$-cell function and Chrebp/Txnip signaling.

Methods: Zucker diabetic fatty rats were treated with racemic Equol (120 mg/kg.BW.d) for 6 weeks. The glucose and lipid metabolism were monitored during the supplementation, and the Chrebp and Txnip expression were measured by using Western blotting. INS-1 cells were incubated with high glucose $(26.2 \mathrm{mM})$ with or without $\mathrm{S}$ Equol (0.1 MM, $1 \mu \mathrm{M}, 10 \mu \mathrm{M})$ for $48 \mathrm{~h}$. Glucose-stimulated insulin secretion (GSIS) was evaluated by radioimmunoassay, and the apoptosis of INS-1 cells was analyzed using Annexin V-FITC/PI and TUNEL assay. The dual luciferase reporter assay, chromatin immunoprecipitation assay and Western-blotting followed by Chrebp small interfering RNAs were utilized to clarify the mechanism of transcriptional regulation of S-Equol on Chrebp/Txnip signaling and the activities of protein kinase A (PKA) and protein phophatase (PP2A) were also detected.
\end{abstract}

Results: In vivo, Equol supplementation delayed the onset of the hyperglycemia and hyperlipemia, ameliorated insulin secretion failure, enhanced GSIS in isolated islets, and significantly reduced Chrebp and Txnip expression in islets. In vitro, S-Equol treatment enhanced GSIS of high glucose cultured INS-1 cell, and reduced apoptosis of INS-1 cells were also observed. Moreover, S-Equol dramatically suppressed Txnip transcription, as evident by the reduction of Txnip protein and mRNA levels and decrease in the Txnip promoter-driven luciferase activity. Meanwhile, S-Equol significantly inhibited Chrebp/Mlx expression and decreased occupancy of Chrebp on the Txnip promoter, and combined with siChrebp, we confirmed that $S$-Equol improvement of insulin secretion was partially through the Chrebp/Txnip pathway. Furthermore, S-Equol significantly decrease nuclear translocation of Chrebp, which was related with the decrease activity of protein kinase A (PKA) and the increase activity of protein phophatase (PP2A).

Conclusions: S-Equol could ameliorate insulin secretion failure, which was dependent on the suppression of Chrebp/Txnip signaling via modulating PKA/PP2A activities.

Keywords: S-Equol, Diabetes, Insulin secretion, Chrebp, Txnip, PKA, PP2A

\footnotetext{
* Correspondence: mi_mantian@sina.com

${ }^{1}$ Research Center for Nutrition and Food Safety, Institute of Military

Preventive Medicine, Third Military Medical University, Chongqing 400038,

People's Republic of China

Full list of author information is available at the end of the article
}

(C) The Author(s). 2020 Open Access This article is distributed under the terms of the Creative Commons Attribution 4.0 International License (http://creativecommons.org/licenses/by/4.0/), which permits unrestricted use, distribution, and reproduction in any medium, provided you give appropriate credit to the original author(s) and the source, provide a link to the Creative Commons license, and indicate if changes were made. The Creative Commons Public Domain Dedication waiver (http://creativecommons.org/publicdomain/zero/1.0/) applies to the data made available in this article, unless otherwise stated. 


\section{Background}

As the prevalence of type 2 diabetes mellitus (T2DM) continues to increase worldwide, identifying dietary factors that can help modulate the insulin sensitivity and insulin secretion process is an important public health and clinical goal for reducing T2DM burden (1). Increasing evidences indicate that dietary soy as well as the isoflavone daidzein or S-Equol could improve glycemic control and insulin responsiveness $(2,3)$, but the conclusions are still uncertain. S-Equol, the end product of daidzein by intestinal bacteria, attracts tremendous attention as its superiority to other isoflavones in its estrogenic and antioxidant activity (4-6). Moreover, results of recent studies (2, 7$10)$ suggest that $S$-Equol may affect glucose and lipid metabolism in ways that were independent of its estrogenic activity, for instance, via increased energy expenditure, decrease release of adipokines, activation of peroxisome proliferator-activated receptor and AMP-activated protein kinase signaling. Thus, the mechanisms whereby S-Equol exerts its beneficial effects should be further elucidated.

Defects in glucose-stimulated insulin secretion (GSIS) by pancreatic $\beta$-cells are central to T2DM risk and progression $(1,3,11)$. Either increased proliferation or decreased cell death in $\beta$-cells directly enhance insulin secretion $(1,3,11)$. Thioredoxin-interacting protein (Txnip), a member of the arrestin family, involves various cellular processes including redox state, inflammation and apoptosis (12-14). Notably, recent studies have also revealed that Txnip is a potent inhibitor of cellular glucose uptake and aerobic glycolysis (15), and plays a particularly key role in hyperglycemia-induced $\beta$ cell apoptosis and diabetes development (13, 14, 16-18). High glucose and diabetes induce Txnip expression, whereas inhibition of Txnip expression or Txnip deficiency protects against pancreatic $\beta$ cell apoptosis and diabetes $(11,19)$. Glucose activates Txnip transcription by recruiting the carbohydrate response element-binding protein (Chrebp) and its obligate transcription partner Mlx to the Txnip promoter $(18,20,21)$. Moreover, recent studies have discovered that the activity of Chrebp is regulated by its phosphorylation status and cellular localization (20, 22-24). High glucose stimulates Chrebp gene expression and also stimulate its translocation from the cytosol to the nucleus, thereby increasing its DNA-binding/transcriptional activity $(25,26)$. In light of Txnip in pathological process in T2DM and the central role of Chrebp in the regulation of Txnip, here we investigated whether S-Equol affects insulin secretion and influences the Chrebp/Txnip signaling both in vivo and in vitro, which would support new evidences to demonstrate the effects and mechanisms of S-Equol on diabetes.

\section{Methods}

Animals and the experimental procedures

A total of 12 male Zucker diabetic fatty (ZDF) rats ( $f a /$ $\mathrm{fa}$ ) and 12 male Zucker lean (ZLR) rats $(\mathrm{fa} /+)$ were purchased from Vital River Laboratories International Inc. (Beijing, China). Rats were scheduled to arrive at 6 weeks of age, and housed two per cage under a 12-h light/dark cycle at controlled temperature conditions with ad libitum access to Purina 5008 chow and water. All animal experiments were approved by the Third Military Medical University Committee for animal research and followed our institutional guidelines for the use of laboratory animals.

Following 2 weeks of adaptation, the rats at 8 weeks of age were randomly assigned to four groups: ZLR rats with no drug treatment (ZLR; $n=6$ ), ZLR rats with Equol supplementation $(E q+Z L R ; n=6)$, ZDF rats with no drug treatment (ZDF; $\mathrm{n}=6$ ), ZDF rats with Equol supplementation $(\mathrm{Eq}+\mathrm{ZDF} ; \mathrm{n}=6)$. Equol (purity $>98 \%$; a racemic mixture of Equol) was purchased from Nanjing Zelang Biotechnology Co. Ltd., China, and Equol were dissolved in distilled water and administered intra-gastrically $(120 \mathrm{mg} /$ $\mathrm{kg} . \mathrm{bw})$ at 9:00 am every day. Body weight, food and water intake were measured every 2 days, blood glucose once weekly, and oral glucose tolerance test (OGTT), serum levels of insulin every 2 weeks. After 6 weeks of treatment, all the rats were sacrificed and total triglyceride (TG), total cholesterol (Tch), low density lipoprotein (LDL), high density lipoprotein (HDL), C-peptide, Glucagon, and $S$ Equol levels were detected. Meanwhile, pancreatic islets (3 in each group) were isolated and cultured overnight for insulin secretion studies as previous described (11); the other pancreatic islets were harvested and stored at $-80^{\circ} \mathrm{C}$ to use in the following experiments of this study.

\section{OGTT}

Rats were fasted for $16 \mathrm{~h}$ prior to OGTT to allow complete drug washout. An oral glucose load of $2 \mathrm{~g} / \mathrm{kg}$ body weight was administered. Blood samples were collected from the tail vein at $0,15,30,60$, and $120 \mathrm{~min}$ after glucose loading. Blood glucose levels were measured using an Accu-check glucose analyzer (Roche, Germany) and overall changes in glucose during OGTT were calculated as areas under the curve (AUC). Meanwhile, using a radioimmunoassay, the blood insulin levels during OGTT at each time point were measured (Linco Research, MO, USA).

\section{Serum indicators measurements}

Serum Tch, TG, HDL, LDL levels were measured by an automatic biochemistry analyzer (Olympus AU5400, Tokyo, Japan). Commercial assay kits were used to determine plasma Glucagon (Rat Glucagon ELISA kit, WAKO, Japan), C-peptide (Rat C-peptide ELISA; ALPCO) levels were measured using ELISA kits. S-Equol was measured by using HPLC method as the previous study (8). 


\section{Glucose-stimulated insulin secretion (GSIS) from isolated islets}

Duplicate samples of three isolated islets were incubated to measure insulin secretion in Dulbecco's modified Eagle's medium (Gibco, USA) with low $(2.8 \mathrm{mM})$ or high $(16.7 \mathrm{mM})$ glucose concentrations for $2 \mathrm{~h}$ at $37^{\circ} \mathrm{C}$. Supernatants were collected and insulin remaining in the islets was extracted with $3 \%$ acetic acid, then insulin was measured using a radioimmunoassay (Linco Research, MO, USA). The total islet insulin content included both the insulin secreted into supernatant and the remaining in the islet. Amplification rate of insulin secretion was calculated as the ratio of insulin release level at high glucose compared with basal glucose stimulation.

\section{Cell culture and the experimental procedures}

Rat insulinoma beta cells (INS-1) were maintained in RPMI 1640 medium (Gibco-Invitrogen, Carlsbad, CA, USA) containing $10 \%$ fetal bovine serum (HyClone, USA), $1 \%$ penicillin/streptomycin, $1 \mathrm{mM}$ sodium pyruvate, $2 \mathrm{mM} \mathrm{L}$-glutamine, $10 \mathrm{mM}$ HEPES, and $0.05 \mathrm{mM}$ 2-mercaptoethanol in a $37^{\circ} \mathrm{C}$ incubator at $5 \% \mathrm{CO}_{2}$. All experiments were performed on the cells when the cells reached $75-85 \%$ confluence. To detect the effect of $S$ Equol on high glucose-treated INS-1 cells, cells were incubated in RPMI medium containing $26.2 \mathrm{mM}$ glucose with or without S-Equol [CAS No: 531-95-3, Dasailu pharmaceutical chiral technology (Shanghai) Co., LTD] at the indicated concentrations $(0.1 \mu \mathrm{M}, 1 \mu \mathrm{M}, 10 \mu \mathrm{M})$ for $48 \mathrm{~h}$.

\section{Glucose-stimulated insulin secretion in INS-1 cells}

INS- 1 cells were seeded $\left(5 \times 10^{5}\right.$ cells/well $)$ in 12 -well plates. After 48-h treatments, INS-1 cells were washed and incubated in KRB-HEPES with 0.1\% BSA for a quiescent period of $90 \mathrm{~min}$. Then, the cells were incubated in KRB-HEPESBSA containing 2.8 or $16.5 \mathrm{mM}$ glucose for $90 \mathrm{~min}$ to respectively measure the basic insulin secretion (BIS) and GSIS. Insulin secreted into the medium was evaluated by the radioimmunoassay. The total cell insulin content was calculated by adding insulin secreted into the supernatant plus that remaining in the cells, with correction by cell number in each group.

\section{Quantitative real-time PCR (qRT-PCR)}

Total RNA was extracted with Trizol buffer and reverse transcribed into cDNA using the BioRT cDNA first strand synthesis kit (Boaosen, Beijing, China) following the manufacturer's recommendations. Quantitative real-time PCR was performed with the Bioeasy SYBR Green Real-time PCR Kit (Boaosen, Beijing, China) on the MyiQ Real-time PCR system (Bio-Rad, USA). All data were analyzed using the mRNA expression of $\beta$-actin as an internal reference. The primer sequences used were as follows: Proinsulin
(PPI; forward 5' - GTGTGGGGAACGTGGTTTCT-3'; reverse $\quad 5^{\prime}$-TGCCAAGGTCTGAAGATCCC -3'); Txnip (forward 5' -TGTGTGAAGTTACTCGTGTCAAA-3'; reverse $5^{\prime}$ - GCAGGTACTCCGAAGTCTGT-3'); $\beta$-actin (forward 5' -ACCAACTGGGACGATATGGAGAAGA-3'; reverse 5' -ACGACCAGAGGCATACAGGGACAA-3').

\section{Western blotting analysis}

Total proteins were extracted with T-PER Protein Extraction kit (Pierce Biotechnology, Inc., Rockford, IL, USA), and nuclear fractions were collected using the Nuclear Protein Extraction Kit (Sigma-Aldrich, MO, USA) according to the manufacturer's instructions. Protein concentrations were determined using the BCA Protein Assay Kit (Beyotime, China). Protein samples were separated by $12 \%$ SDSPAGE and transferred to PVDF membranes (Millipore, Billerica, MA, USA). After blocking with 5\% BSA at room temperature for $1 \mathrm{~h}$, the membranes were incubated overnight at $4{ }^{\circ} \mathrm{C}$ with the following primary antibodies: Glucose transporter 2 (Glut2; Santa Cruz Biotechnology, CA, USA),Uncoupling protein-2 (UCP-2; Abcam, Cambridge, UK), Cherbp (Novus, Oakville, Canada) and Txnip (Novus, Oakville, Canada). $\beta$-Actin (Beyotime, Beijing, China) was used as a loading control for total proteins and Lamin A (Cell Signaling Technology, MA, USA) for nuclear proteins. Then membranes were washed and incubated in appropriate secondary antibody for $1 \mathrm{~h}$. The target proteins were detected using chemiluminescence system (Pierce Biotechnology), and the immunoblots were quantified by densitometry using Bio-Rad Quantity One 4.4.0 software (Bio-Rad Lab., Hercules, CA, USA).

\section{Annexin V-FITC/PI assay}

Apoptosis was detected using an Annexin V-FITC/PI detection kit (Bipec Biopharma Corp., USA) according to the manufacturer's instructions. Briefly, cells were harvested, washed and resuspended in binding the buffer. Then cells were mixed with AnnexinV-FITC and propidium iodide (PI) for $15 \mathrm{~min}$ at room temperature in the dark. Fluorescence was detected by flow cytometry (FACS-400, USA). The percentage of apoptosis was reflected by the number of Annexin $\mathrm{V}(+) / \mathrm{PI}(-)$ cells relative to the number of Annexin $\mathrm{V}(+) / \mathrm{PI}(+)$ cells.

\section{Terminal deoxynucleotidyl transferase dUTP nick end labeling (TUNEL) assay}

Apoptotic INS-1 cells were also studied using TUNEL assay. INS-1 cells were seeded on coverslips. After treatment, cells were washed with PBS, fixed in $4 \%$ paraformaldehyde, permeabiliz ed. with $0.1 \%$ Triton X-100 and blocking with 5\% BSA. Fluorescein in Situ Cell Death Detection kit (Millipore cat. \#S7110, Billerica, MA, United States) was used according to the manufacturer's protocol. Six different fields in each coverslip and six 
samples per group were used to collect the images and the quantification of TUNEL positive cells and Apoptotic rate. The images were obtained using a confocal microscope (Zeiss LSM 510 META; Carl Zeiss, Oberkochen, Germany).

\section{Txnip promoter activity assay}

INS-1 cells were plated in 96-well plates and incubated until the cells reached $80 \%$ confluence. The rat Txnip promoter-luciferase reporter (-500 to $279 \mathrm{bp}$ ) plasmid constructs were constructed as the previous study (27). INS-1 cells were transfected with the Txnip promoterluciferase reporter plasmid or the empty vector (internal control) using Lipofectamine 2000 (Invitrogen, Carlsbad, CA, USA) following the manufacturer's protocol. After transfection, cells were incubated in high glucose conditions with or without $S$-Equol for $48 \mathrm{~h}$. Luciferase activity was analyzed using the Dual-Luciferase Assay Detection kit (Promega, Madison, WI, USA) with a luminometer microplate reader (Molecular Devices, USA) according to the manufacturer's instructions. Normalized luciferase values were expressed as the percentage relative to the control group.

\section{Chromatin immunoprecipitation (ChIP) assay}

ChIP assay was performed using a ChIP assay kit (Cell Signaling Technology) following the manufacturer's instructions. After the indicated treatment, cells were harvested. After crosslinking the chromatin in $1 \%$ formaldehyde at $37^{\circ} \mathrm{C}$ for $10 \mathrm{~min}$ and neutralizing with glycine for $5 \mathrm{~min}$ at room temperature, the cells were washed with cold PBS and lysed in sodium dodecyl sulfate lysis buffer. Subsequently, the chromatin was sonicated to generate an average length of 150-900 bp DNA fragments. Immunoprecipitation of crosslinked protein/DNA was performed overnight at $4{ }^{\circ} \mathrm{C}$ with the following antibodies: Chrebp (Santa Cruz Biotechnology) and normal rabbit IgG (as a negative control; Cell Signaling Technology). The purified DNA fragments were quantified by PCR using primers 5 ' -CGCACCCGAACAAC AACCAT-3' (forward) and 5' - AAGCGGGAGCCGGA AACGG-3' (reverse) to determine the binding of Cherbp to Txnip promoter.

\section{Chrebp small interfering RNA and transfection}

Predesigned Chrebp siRNA and control scramble siRNA (as the negative control) were synthesized by Invitrogen. The stealth RNAi molecules $(40 \mathrm{nM})$ were transfected into INS-1 cells using Lipofectamine 2000 according to the manufacturer's instructions. The ability of the stealth RNAi oligonucleotide to knockdown Chrebp expression was analyzed by Western- blotting using the total cell extract.
Detection of the activities of protein kinase a (PKA) and protein phosphatase 2A (PP2A)

INS-1 cells were collected and cell lysate was used to detect PKA activity using a PKA Kinase Activity Assay Kit (Abcam, Cambridge, UK) according to the manufacturer's instructions. PP2A activity was measured using a Serine/Threonine Phosphatase Assay Kit (Promega, Madison, USA) following the manufacturer's instructions.

\section{Statistics}

All experiments were performed at least three times. Data were presented as the mean \pm standard deviation (SD). Different groups were compared by one-way analysis of variance (ANOVA) followed by Tukey-Kramer post hoc tests for multiple comparisons. A value of $P<$ 0.05 was considered statistical significant.

\section{Results}

S-Equol improves hyperglycemia and hyperlipemia in ZDF rats

To determine how S-Equol involved in the development of diabetic phenotypes in ZDF rats, we observed the effects of Equol (120 mg/kg.bw.d) treatment on body weight, water intake, food intake, serum glucose level, and serum lipid profile. During the first 3 weeks of the supplementation, relatively lower body weights were seen in ZDF rats with Equol, when compared to ZDF rats without Equol feeding (Fig. 1a). However, the difference in body weight were disappeared in the following 3 weeks, and after 6 weeks treatment, the body weights of ZDF rats fed with Equol kept increasing while ZDF rats without Equol turns to lose weight (Fig. 1a). Meanwhile, the amount of water intake in ZDF rats with Equol was less than that of ZDF rats without Equol in the whole supplementation period (Fig. 1b), but there were no differences in the amount of food intake (Fig. 1c). Serum glucose levels of ZDF rats given Equol were significantly reduced compared to the ZDF rats in the whole supplementation period of this study (Fig. 1d). Furthermore, OGTT revealed significant amelioration of glucose metabolism in ZDF rats with Equol treatment (Fig. 1e-h). The changes in Tch, TG, LDL-c, HDL-c were shown in Fig. 1i-l, which indicated that Equol supplementation led to a significant reduction of Tch and LDL in ZDF rats. These results suggest that Equol supplementation improves glucose and lipid metabolism and enhances glucose tolerance in ZDF rats.

Additionally, as S-Equol was the bioactive form of Equol, we detected the plasma level of S-Equol by using HPLC assay. After the 6 weeks' supplementation, plasma levels of $S$-Equol in Equol supplemented ZDF rats reached at $7.04 \pm 1.27 \mu \mathrm{M}$ and $5.31 \pm 0.86 \mu \mathrm{M}$ in ZLR rats, whereas only $0.53 \pm 0.24 \mu \mathrm{M}$ and $0.47 \pm 0.31 \mu \mathrm{M}$ in the ZLR rats and ZDF rats without Equol supplementation. 


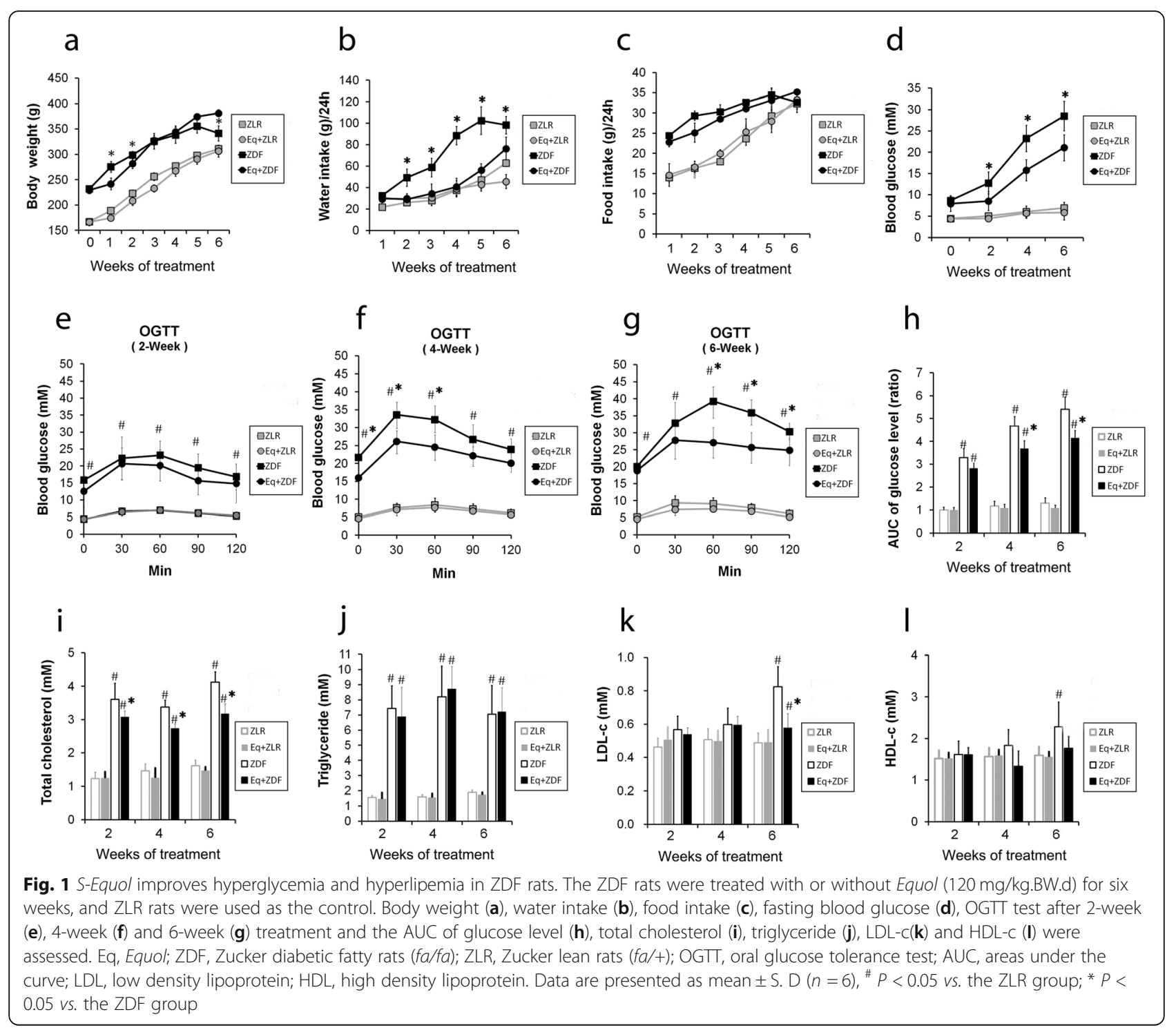

\section{$S$-Equol supplementation improves insulin secretion in ZDF rats}

ZDF rats showed enhanced insulin secretion after 2 weeks treatment, and with the progression of obesity, insulin secretion decreased after 6 weeks treatment (Fig. 2a). Compared with ZDF rats, marginally significant higher levels of serum insulin were observed in ZDF rats supplemented with Equol after 2, 4 and 6 weeks' treatment (Fig. 2a) and significant higher levels of C-peptide were detected in Equol supplemented ZDF rats after 6 weeks treatment (Fig. 2b). Meanwhile, slightly reduction of serum glucagon were detected in Equol supplemented ZDF rats after 6 weeks treatment (Fig. 2c). Furthermore, during OGTT tests, serum insulin levels at each time point were higher in Equol-treated-ZDF rats compared with those without Equol (Fig. 2d). To evaluate the effect of Equol on insulin secretion ex vivo, isolated pancreatic islets were used to determine GSIS. As shown in Fig. 2 e-f, insulin secretion from ZDF rat islets in response to high glucose was blunted, but was improved by Equol. These results suggest that Equol ameliorates insulin secretion failure in ZDF rats both in vivo and ex vivo.

\section{S-Equol treatment alleviates GSIS in high-glucose cultured INS-1 cells}

To further identify the effects of S-Equol on insulin secretion, serial experiments with INS-1 cells cultured in high glucose were done. Insulin secretion in response to 5 or $25 \mathrm{mM}$ of glucose was measured from INS-1 cells (Fig. 3a). As previously reported (3), insulin release in response to 5 or $25 \mathrm{mM}$ of glucose was drastically reduced for cells cultured at high glucose as compared with the control cells. When S-Equol (0.1, 1 or $10 \mu \mathrm{M})$ was included during the culture of INS-1 cells with high glucose, insulin secretion 


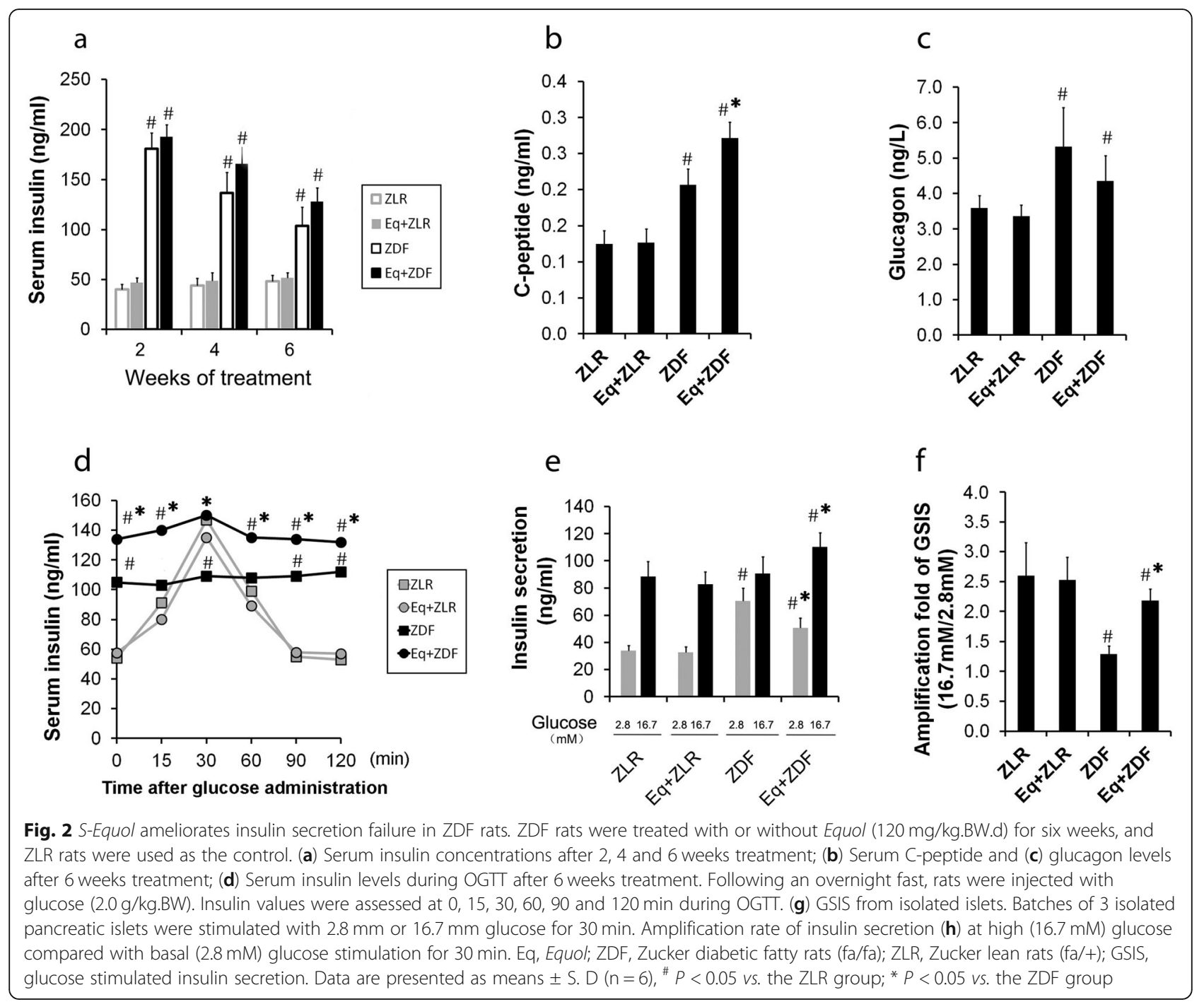

in response to 5 or $25 \mathrm{mM}$ glucose significantly increased as compared with the cells cultured in the absence of $S$ Equol. Meanwhile, to further analyze specific effects on insulin production, real-time PCR analysis for PPI mRNA (Fig. 3b) and Western blotting analysis for Glut2 and UCP2 were performed (Fig. $3 \mathrm{c}-\mathrm{d}$ ). Compared with the high glucose cultured INS-1 cells, INS-1 cells treated with $S$ Equol showed significant increase in their cellular PPImRNA content and Glut2 protein and decrease in UCP-2 protein (Fig. 3b-d).

\section{$S$-Equol increases cell proliferation and inhibits apoptosis of INS-1 cells cultured in high glucose}

The deterioration of GSIS in $\beta$-cells exposed to elevated levels of glucose is connected with the loss of $\beta$-cell mass $(1,3)$. In the present study, we sought to determine whether the observed beneficial effect of S-Equol on improved secretion response was related to the increased cell proliferation or the reduced apoptosis of INS-1 cells.
As the results of MTT tests, S-Equol $(0.1,1,10 \mu \mathrm{M})$ treatment had improved cell viability of INS-1 cells cultured in high glucose with $78.55,88.93$ and $64.78 \%$ respectively, compared with $42.04 \%$ in INS-1 cells cultured in high glucose in the absence of S-Equol. Next, we examined the presence of apoptosis in normal glucose and high glucose-treated cells with or without S-Equol. Both TUNEL assay and Annexin- $V$ staining showed significantly increase apoptotic cells by glucotoxicity and decreased by treatment with S-Equol (Fig. 3e-f).

\section{S-Equol supplementation suppresses Chrebp/Txnip signaling in vivo and in vitro}

Txnip has been reported to play central roles in diabetes progression particularly in relation to $\beta$-cell apoptosis (11). In the present study, S-Equol was proved to reduce INS-1 cell apoptosis. To verify the relation between $S$ Equol and Txnip, with western blotting analysis, we found that $S$-Equol significantly inhibited Txnip increase 


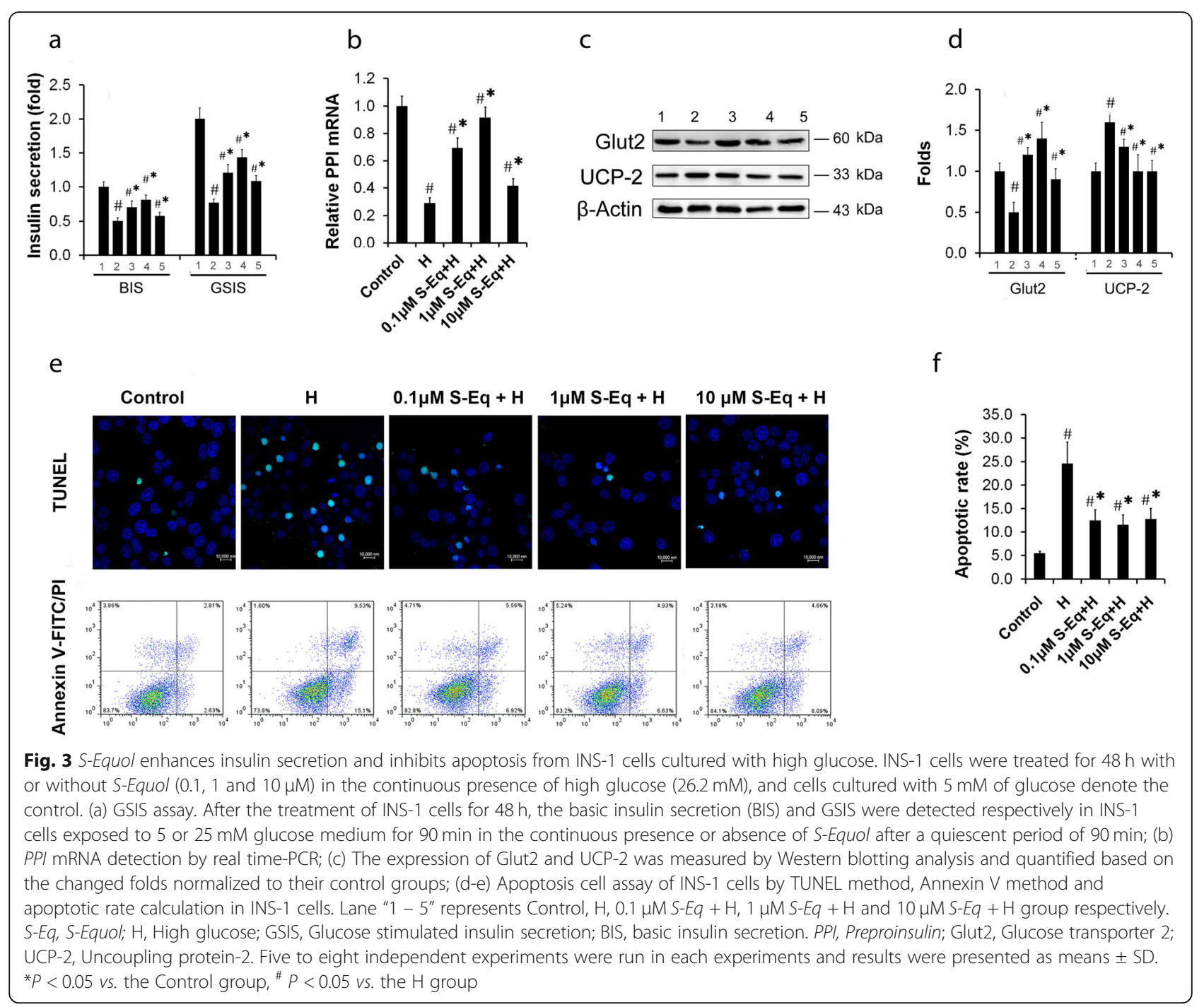

in ZDF rats (Fig. 4). Similar results were seen in Txnip protein and mRNA levels in S-Equol administrated high glucose cultured INS-1 cells (Fig. 5 a-c). In addition, we found that this $S$-Equol inhibition of the glucose-induced Txnip over expression occurred at the transcriptional levels as demonstrated by the decrease in the Txnip promoter-driven luciferase activity (Fig. 5d).

The transcription complex Chrebp/Mlx is determinant for the induction of Txnip genes $(15,20,21,26)$. As shown in Fig. 4a-b, Chrebp and Mlx protein in ZDF rats were partially inhibited in ZDF rats and in INS-1 cells with Equol treatment compared with those without Equol. Moreover, we found that S-Equol treatment decreased Chrebp occupancy of the Txnip promoter in vitro as demonstrated by ChIP assay, whereas the IgG control showed nearly no enrichment confirming the specificity of these ChIP assays (Fig. 5 e). Furthermore, transfection of INS-1 cells with Chrebp small interfering RNAs (Fig. $5 \mathrm{f}$-h) not only resulted in effective Chrebp knockdown, but also led to a dramatic reduction in Txnip expression, further confirming the important role Chrebp plays in S-Equol regulating Txnip transcription. Taken together, S-Equol suppresses Chrebp/Txnip signaling in vivo and in vitro.

\section{$S$-Equol regulates Chrebp/Txnip signaling via modulating PKA/PP2A activities}

Nuclear translocation of Chrebp is one of the important processes in the glucose activation of Txnip gene transcription $(15,21,28)$. To determine whether S-Equolmediated inhibition in Txnip gene expression was correlated to a decrease in nuclear Chrebp content, Western blot analysis was performed using nuclear protein extracts from INS-1 cells cultured in high glucose with the presence or absence of S-Equol (Fig. 6a). Incubation of INS-1 cells with glucose led to a 3.4-fold increase in Chrebp content in the nucleus. In contrast, after S-Equol treatment, the Chrebp translocation to the nucleus was partially inhibited. 

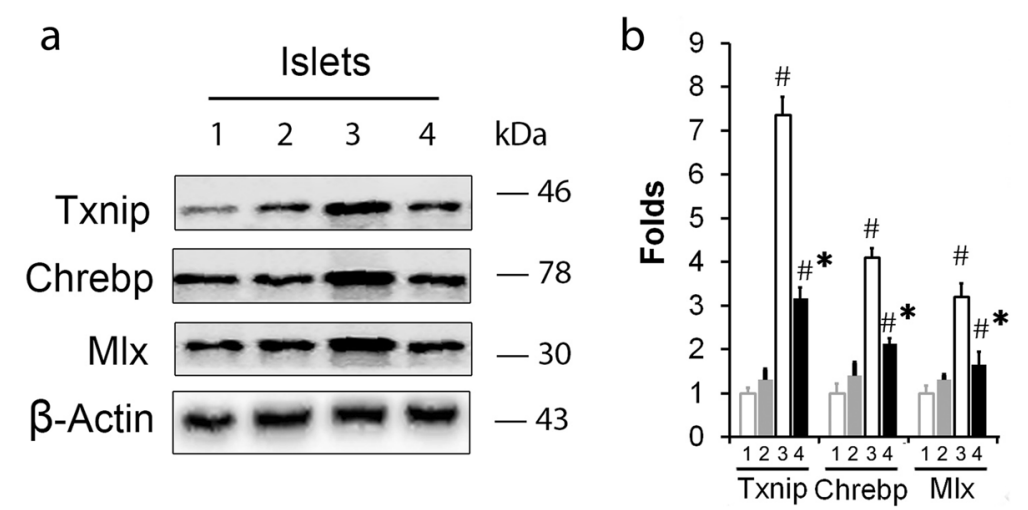

Fig. 4 S-Equol regulates Chrebp /Txnip signaling in ZDF rats. (a) Western blotting to determine Txnip, Chrebp and Mlx expression in ZDF rats with or without Equol (120 mg/kg.BW.d). (b) Densitometric quantification of the relative folds of the proteins /actin ratio in ZDF rats was shown in bar graph. ZDF, Zucker diabetic fatty rats (fa/fa); ZLR, Zucker lean rats (fa/t). Lane "1 - 4" represents ZLR, Eq + ZLR, ZDF and Eq + ZDF group respectively. Three independent experiments were run and results were presented as means \pm SD. ${ }^{\#} P<0.05$ vs. the Control group, ${ }^{*} P<0.05$ vs. ZDF group

Translocation of Chrebp into the nucleus has been shown to require a number of dephosphorylation steps, in particular dephosphorylation of Ser ${ }^{196}$ by PP2A (19). We therefore investigated the activity of PP2A, and indeed found a clear decline in high-glucose cultured INS-1 cells with S-Equol treatment (Fig. 6b). Moreover, it was proposed that Chrebp/Mlx heterodimer forming and DNA binding resulted from dephosphorylation Ser $^{666}$ (phosphorylated by

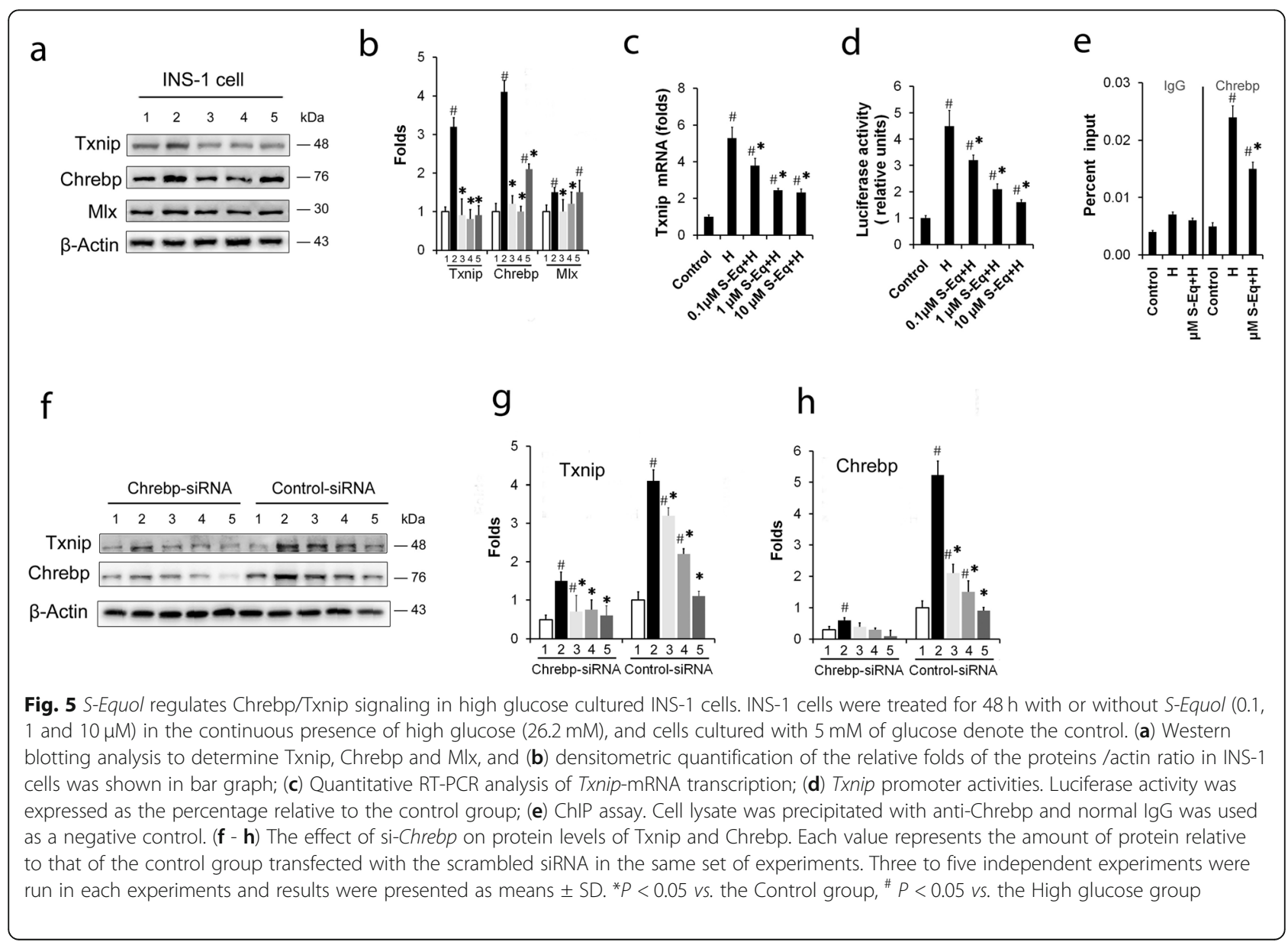


a
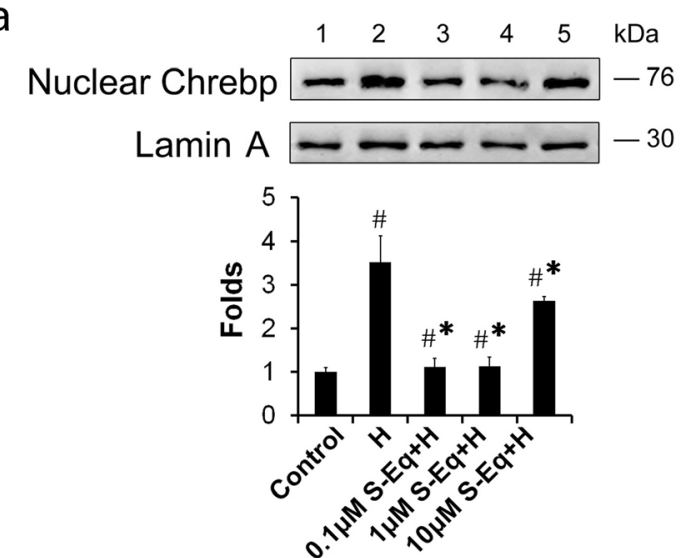

b

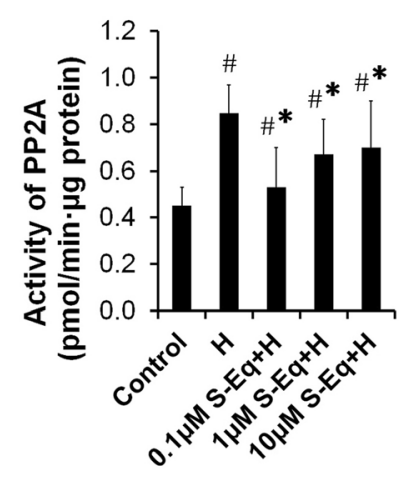

C

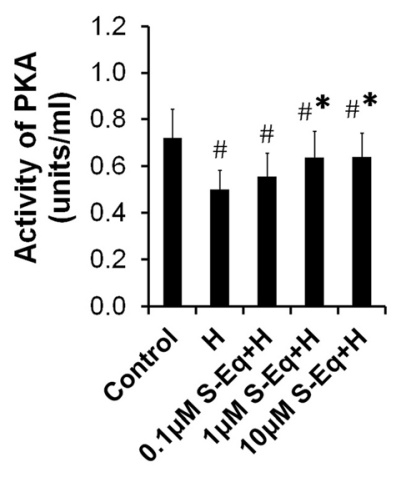

Fig. 6 S-Equol modulates PKA/PP2A activities in high glucose cultured INS-1 cells. INS-1 cells were treated for $48 \mathrm{~h}$ with or without S-Equol (0.1, 1 and $10 \mu \mathrm{M})$ in the continuous presence of high glucose $(26.2 \mathrm{mM})$, and cells cultured with $5 \mathrm{mM}$ of glucose denote the control. (a) Western blotting analysis of nuclear Chrebp; (b) The PP2A and (c) PKA activities. H, High glucose; S-Eq, S-Equol; Lane "1 - 5" represents Control, H), $0.1 \mu \mathrm{M}$ $\mathrm{S}-\mathrm{Eq}+\mathrm{H}, 1 \mu \mathrm{MS}$-Eq $+\mathrm{H}$ and $10 \mu \mathrm{M}$ S-Eq $+\mathrm{H}$ group respectively; PP2A,protein phophatase 2A; PKA, protein kinase A. Three to five independent experiments were run in each experiments and results were presented as means \pm SD. ${ }^{*} P<0.05$ vs. the Control group, ${ }^{\#} P<0.05$ vs. the High glucose group

PKA) (28), directly affecting the transcriptional efficacy. We therefore determined the activity of PKA and results showed that S-Equol significantly enhance the PKA activity in high-glucose cultured INS-1 cells (Fig. 6c). Overall, these results suggested that $S$-Equol-mediated inhibition of Chrebp/Txnip signaling was partially related to modulate the PKA and PP2A activities.

\section{Discussion}

In the present study, we confirmed the anti-diabetic activity of S-Equol in ZDF rats, a type 2 diabetic animal model, and clarified the regulatory mechanism of insulin secretion by S-Equol in relation to influence the Chrebp/ Txnip signaling via modulating the activities of PP2A and PKA in high glucose cultured INS-1 cells. The current results provide a novel mechanism for elucidating the anti-diabetic effect of $S$-Equol.

$S$-Equol is the main active product of daidzein metabolism, which is produced by specific microflora in the gut. "Equol hypothesis", proposed by Setchell et al. (6), states that Equol producers (organisms possessing microbiota that can produce $S$-Equol) receive maximal health benefits from soy intake. S-Equol has a stronger estrogenic effect than daidzein, leading to the relief of menopausal symptoms and the prevention of prostate cancer, which supports the Equol hypothesis (4, 6, 29). Notably, recent evidences, including $S$-Equol supplementation decreased glycated hemoglobin concentrations in non-S-Equol-producing subjects $(8,9)$ and racemic Equol administration suppressed hyperglycemia in ob/ob mice (10), alloxaninduced mice and STZ-induced rats (3), all support that $S$-Equol was a powerful metabolic regulators even in Equol non-producers and the potential mechanism was beyond the estrogenic effect. In this study, Equol supplementation were also identified to substantially improve diabetic phenotypes in ZDF rats. Firstly, it is known that the weight loss and the increase of water intake that occurs unintentionally were warning signs of aggravating diabetes (30). ZDF rats treated with Equol still kept gaining weight whereas the weight loss occurred in ZDF rats at 14th week age. Meanwhile, Equol supplementation restricted the water intake of ZDF rats. Secondly, Equol significantly reduced the increase of glucose levels of ZDF rats in the whole treatment period. Admittedly, the concentrations of glucose of ZDF rats treated with Equol remained to be higher than normal. This might partially attributed to the feature of the ZDF rat, which has a missense mutation in the gene coding the leptin receptor $(\mathrm{fa} / \mathrm{fa})$ and spontaneously develops T2DM with severe diabetic phenotypes including higher glucose levels (31). Thirdly, Equol improved the lipid profile of ZDF rats. In line with our results, lower prevalence of dyslipidemia was observed in Equol producers (32) and reduced lipid levels were also found in Equol treated ob/ob mice (33), and further studies revealed the anti-hyperlipemia effect of Equol was correlated with the inhibition of hepatic lipid synthesis and the modulation of intestinal lipid absorption. As mounting evidences demonstrated Equol given regularly was capable to improve symptoms of diabetes and be a supportive therapy in the treatment of T2DM, further study to unravel the underlying mechanism were need.

Defects in insulin secretion are a critical determinant of the risk and progression of T2DM $(1,11)$. The total number of $\beta$-cells (related to proliferation and survival) and glucose response in individual $\beta$-cells are responsible for insulin secretion (1). In this study, we showed 
that S-Equol increased glucose-stimulated insulin secretion in vivo and in vitro, which might correlated with both $\beta$-cell proliferation increase and $\beta$-cell apoptosis inhibition. In line with our results, previous studies (2, 3, 10) also identified that S-Equol could prevent oxidative stress-induced cell death in INS- 1 cells and enhance $\beta$ cell proliferation in STZ-induced diabetic rats. Besides inhibiting loss of $\beta$-cells, S-Equol was also found to play a role in enhancing insulin synthesis as evident by significantly elevated C-peptide in ZDF rats and increased cellular PPI mRNA in high-glucose cultured INS-1 cells. Interestingly, concerning the effects on PPI mRNA transcription in INS-1 cells, S-Equol at high dosage $(10 \mu \mathrm{M})$ was not better than that at low dosage $(0.1,1 \mu \mathrm{M})$. Insulin synthesis was regulated at both the transcriptional and translational level (34), and PPI mRNA transcription was regulated by many trans-activators including paired box gene 6, pancreatic and duodenal homeobox-1, MafA, and $\beta-2 /$ Neurogenic differentiation 1 . The underlying mechanism of the insulin synthesis modulation by $S$-Equol might be complicated and further investigations were needed to explain these phenomena.

Txnip is a protein with multifunctional roles in diverse cellular responses such as proliferation, apoptosis and differentiation (14). Recently, Txnip was proved to be essential for glucotoxicity-induced $\beta$-cell death, whereas lack of Txnip promotes endogenous $\beta$-cell survival and prevents type 1 and type 2 diabetes $(11,21)$. In this study, we found that the content of Txnip protein was significantly reduced by $S$-Equol in ZDF rats and high glucose cultured INS-1 cells. Consistent with our results, some other soy derived isoflavones including genistein (35) and Biochanin A (36), and resveratrol $(37,38)$ have also been proved to inhibit Txnip expression, however, there was no further investigation about the mechanisms. Generally, the cellular Txnip level is regulated at the transcriptional level, post-translational level and degradation rate (27). In the present study, Txnip mRNA levels dropped by over $50 \%$ and significantly decrease in the Txnip promoter transcription activity were determined in high-glucose cultured INS-1 cells with S-Equol treatment, which suggests that $S$-Equol mediated drop in Txnip levels is likely due to transcriptional repression of Txnip.

Glucose-induced Txnip expression is mediated via a nonpalindromic E-box motif consisting of a repeat of CACGAG sequences in the Txnip promoter that serves as the binding site for the Chrebp $(21,26,28)$. The basic helixloop-helix transcription factor Chrebp has been recognized as the main transcription factor mediating glucose-induced gene expression in liver as well as in $\beta$-cells, whereas its paralog, Mondo A, has been shown to do the same in muscle, and a recently discovered isoform, Chrebp- $\beta$, has been described in adipocytes (23). In the present study, as evidenced by a significant Txnip reduction concomitant with Chrebp knocking down by si-chrebp, the relationship between Chrebp and Txnip was confirmed. Furthermore, the decreased robust Chrebp/Mlx protein content and Chrebp occupancy on the Txnip promoter were observed in high-glucose cultured INS-1 cells with S-Equol treatment, which indicates that $S$-Equol could inhibit the transcription factor Chrebp binding to the E-box of the Txnip promoter.

Translocation of ChREBP from the cytosol to the nucleus in response to elevated glucose concentrations has been documented in both liver (39) and clonal pancreatic $\beta$-cells $(21,25,40,41)$ and is likely to be a key regulatory step in the activation of downstream genes (28). Our study demonstrates that $S$-Equol not only decreases Chrebp expression but also reduces the translocation of Chrebp from the cytosol to the nucleus. Previous studies have proved that glucose-dependent Chrebp nuclear translocation is subject to a series of phosphorylation/dephosphorylation reactions. In the liver, while in fasting and in low glucose conditions, Chrebp is phosphorylated by PKA on $\mathrm{S}^{196}$ and $\mathrm{T}^{666}$ and by AMP-activated protein kinase on binding; by contrast, in high glucose conditions, PP2A activation by xylulose 5-phosphate induces dephosphrylation of $S^{196}$, $\mathrm{T}^{666}$, and $\mathrm{S}^{568}$, nuclear import and DNA binding (19). Moreover, recent studies also confirmed that, in the $\beta$-cell, elevated glucose concentrations are partially associated with PP2A inhibition and the elevation of the intracellular cAMP levels and PKA signaling $(3,28)$. In the present study, S-Equol was proved to activate PKA and inhibit PP2A in high-glucose cultured INS-1 cells, which might directly affect the Chrebp/Mlx heterodimers forming and DNA binding and thereby reduce the Txnip transcription. Consistent with that, Hiroko et al. (3) had reported that treatment with a PKA inhibitor diminished S-Equol-mediated effects on $\beta$-cell proliferation and glucose-stimulated insulin secretion in streptozotocin-induced hyperglycemia rats, and oxidative stress (alloxan)-induced cell death. These results further demonstrated that S-Equol ameliorates insulin secretion failure through Chrebp/Txnip signaling via modulating PKA/PP2A activities.

\section{Conclusion}

To our knowledge, this is the first report to illustrate the relationship between Txnip and the anti-diabetic effects of $S$-Equol. Moreover, in the present study, the serum $S$ Equol concentration after 6 weeks of $120 \mathrm{mg} / \mathrm{kg}$ racemic Equol gavage in ZDF rats was about $7 \mathrm{mmol} / \mathrm{L}$, and the effective dose observed in INS-1 cells was ranged from 0.1 to $10 \mu \mathrm{M}$. Given that blood S-Equol concentrations are $>1 \mathrm{mM}$ in some $S$-Equol producers $(6,29)$, our results further confirmed that taking an S-Equol supplement has the potential to prevent T2DM and relieve the prediabetic symptoms. 


\section{Abbreviations}

AUC: Areas under the curve; BIS: Basic insulin secretion; ChIP: Chromatin immunoprecipitation; Chrebp: Carbohydrate response element-binding protein; Eq: Equol; GSIS: Glucose-stimulated insulin secretion; HDL: High density lipoprotein; LDL: Low density lipoprotein; OGTT: Oral glucose tolerance test; PI: Propidium iodide; PKA: Protein kinase A; PP2A: Protein phophatase; PPI: Preproinsulin; QRT-PCR: Quantitative real-time PCR; T2DM: Type 2 diabetes mellitus; Tch: Total cholesterol; TG: Total triglyceride; TUNEL: Terminal deoxynucleotidyl transferase dUTP nick end labeling; Txnip: Thioredoxin-interacting protein; ZDF: Zucker diabetic fatty; ZLR: Zucker lean

\section{Acknowledgements}

Dr. Yujie Huang and Dr. Qicheng Zhou, Army medical University, are acknowledged for their technological suggestions in conducting experiments.

\section{Authors' contributions}

Conceptualization, Ka Chen; Methodology, Li Wang, Hedong Lang, Kai Liu and Ka Chen; Validation, Ka Chen, Hedong Lang and Yong Zhou.; Formal Analysis, Li Wang, Kai Liu and Ka Chen; Data Curation, Li Wang, Hedong Lang and Ka Chen; Writing - Original Draft Preparation, Ka Chen and Hedong Lang; Writing - Review \& Editing, Hedong Lang and Mantian Mi; Supervision, Mantian Mi; Project Administration, Mantian Mi; Funding Acquisition, Ka Chen, Yong Zhou, and Mantian Mi.

\section{Funding}

This study was supported by the National Natural Science Foundation of China (81102128), the Basic Science and Frontier Technology Research Project of Chongqing (CSTC2017jcyjAX0305) and the open Project of Key laboratory of Electromagnetic Radiation Medical Protection of Ministry of Education, Army Medical University (2017DCKF002).

\section{Availability of data and materials}

All data generated or analyzed during this study are included in this published article or are available from the corresponding author on reasonable request.

\section{Ethics approval and consent to participate}

This study was approved by the ethics committee of Army Medical University.

\section{Consent for publication}

The authors consent to the publication of the data.

\section{Competing interests}

The authors declare they have no competing interests.

\section{Author details}

${ }^{1}$ Research Center for Nutrition and Food Safety, Institute of Military Preventive Medicine, Third Military Medical University, Chongqing 400038, People's Republic of China. ${ }^{2}$ Department of Clinic Nutrition, People's Hospital of Chongqing Banan District, Chongqing 401320, People's Republic of China.

Received: 29 April 2019 Accepted: 2 January 2020

Published online: 14 January 2020

\section{References}

1. Holmes D. Diabetes: why beta cells fail in T2DM. Nat Rev Endocrinol. 2017: 13(8): 440.

2. Horiuchi H, Harada N, Adachi T, Nakano Y, Inui H, Yamaji R. S-Equol Enantioselectively activates CAMP-protein kinase a signaling and reduces Alloxan-induced cell death in INS-1 pancreatic-cells. J Nutr Sci Vitaminol (Tokyo). 2014;60(4):291-6.

3. Horiuchi H, Usami A, Shirai R, Harada N, Ikushiro S, Sakaki T, et al. S-Equol activates CAMP signaling at the plasma membrane of INS-1 pancreatic $\beta$ cells and protects against Streptozotocin-induced hyperglycemia by increasing $\beta$-cell function in male mice. J Nutr. 2017;147(9):1631-9.

4. Muthyala RS, Ju YH, Sheng S, Williams LD, Doerge DR, Katzenellenbogen BS, et al. Equol, a natural estrogenic metabolite from soy isoflavones: convenient preparation and resolution of $\mathrm{R}$ - and $\mathrm{S}$-equols and their differing binding and biological activity through estrogen receptors alpha and beta. Bioorg Med Chem. 2004;12(6):1559-67.

5. Nishimura Y, Mabuchi K, Takano A, Hara Y, Negishi H, Morimoto K, et al. Sequol exerts estradiol-like anorectic action with minimal stimulation of estrogen receptor-alpha in Ovariectomized rats. Front Endocrinol. 2017;8:281.

6. Setchell KD, Clerici C. Equol: history, chemistry, and formation. J Nutr. 2010; 140(7):1355s-62s.

7. Minatoya M, Kutomi G, Asakura S, Otokozawa S, Sugiyama Y, Nagata Y, et al. Equol, adiponectin, insulin levels and risk of breast cancer. Asian Pac J Cancer Prev : APJCP. 2013;14(4):2191-9.

8. Sakane N, Kotani K, Tsuzaki K, Takahashi K, Usui T, Uchiyama S, et al. Equol producers can have low leptin levels among prediabetic and diabetic females. Ann Endocrinol. 2014;75(1):25-8.

9. Shor D, Sathyapalan T, Atkin SL, Thatcher NJ. Does equol production determine soy endocrine effects? Eur J Nutr. 2012;51(4):389-98.

10. Cheong SH, Furuhashi K, Ito K, Nagaoka M, Yonezawa T, Miura Y, et al. Antihyperglycemic effect of equol, a daidzein derivative, in cultured L6 myocytes and Ob/Ob mice. Mol Nutr Food Res. 2014;58(2):267-77.

11. Yoshihara E, Fujimoto S, Inagaki N, Okawa K, Masaki S, Yodoi J, et al. Disruption of TBP-2 ameliorates insulin sensitivity and secretion without affecting obesity. Nat Commun. 2010;1:127.

12. Li X, Kover KL, Heruth DP, Watkins DJ, Guo Y, Moore WV, et al. Thioredoxininteracting protein promotes high-glucose-induced macrovascular endothelial dysfunction. Biochem Biophys Res Commun. 2017;493(1):291-7.

13. Oslowski CM, Hara T, O'Sullivan-Murphy B, Kanekura K, Lu S, Hara M, et al. Thioredoxin-interacting protein mediates ER stress-induced beta cell death through initiation of the inflammasome. Cell Metab. 2012;16(2):265-73.

14. Chong CR, Chan WP, Nguyen TH, Liu S, Procter NE, Ngo DT, et al. Thioredoxin-interacting protein: pathophysiology and emerging pharmacotherapeutics in cardiovascular disease and diabetes. Cardiovasc Drugs Ther. 2014;28(4):347-60.

15. Yu FX, Chai TF, He H, Hagen T, Luo Y. Thioredoxin-interacting protein (Txnip) gene expression: sensing oxidative phosphorylation status and glycolytic rate. J Biol Chem. 2010;285(33):25822-30.

16. Sullivan WJ, Mullen PJ, Schmid EW, Flores A, Momcilovic M, Sharpley MS, et al. Extracellular matrix remodeling regulates glucose metabolism through TXNIP destabilization. Cell. 2018;175(1):117-32.

17. Waldhart AN, Dykstra H, Peck AS, Boguslawski EA, Madaj ZB, Wen J, et al. Phosphorylation of TXNIP by AKT mediates acute influx of glucose in response to insulin. Cell Rep. 2017;19(10):2005-13.

18. Wu N, Zheng B, Shaywitz A, Dagon Y, Tower C, Bellinger G, et al. AMPKdependent degradation of TXNIP upon energy stress leads to enhanced glucose uptake via GLUT1. Mol Cell. 2013;49(6):1167-75.

19. Chen J, Jing G, Xu G, Shalev A. Thioredoxin-interacting protein stimulates its own expression via a positive feedback loop. Mol Endocrinol. 2014;28(5): 674-80.

20. Richards P, Ourabah S, Montagne J, Burnol AF, Postic C, Guilmeau S. MondoA/ChREBP: the usual suspects of transcriptional glucose sensing; implication in pathophysiology. Metab Clin Exp. 2017;70:133-51.

21. Cha-Molstad H, Saxena G, Chen J, Shalev A. Glucose-stimulated expression of Txnip is mediated by carbohydrate response element-binding protein, p300, and histone H4 acetylation in pancreatic beta cells. J Biol Chem. 2009; 284(25):16898-905.

22. Vijayakumar A, Aryal P, Wen J, Syed I, Vazirani RP, Moraes-Vieira PM, et al. Absence of carbohydrate response element binding protein in adipocytes causes systemic insulin resistance and impairs glucose transport. Cell Rep. 2017;21(4):1021-35.

23. Abdul-Wahed A, Guilmeau S, Postic C. Sweet sixteenth for ChREBP: established roles and future goals. Cell Metab. 2017;26(2):324-41.

24. Dentin R, Benhamed F, Pegorier JP, Foufelle F, Viollet B, Vaulont S, et al. Polyunsaturated fatty acids suppress glycolytic and lipogenic genes through the inhibition of ChREBP nuclear protein translocation. J Clin Invest. 2005; 115(10):2843-54.

25. Davies MN, O'Callaghan BL, Towle HC. Glucose activates ChREBP by increasing its rate of nuclear entry and relieving repression of its transcriptional activity. J Biol Chem. 2008;283(35):24029-38.

26. Havula E, Hietakangas V. Sugar sensing by ChREBP/Mondo-mlx-new insight into downstream regulatory networks and integration of nutrient-derived signals. Curr Opin Cell Biol. 2017:51:89-96.

27. Kibbe C, Chen J, Xu G, Jing G, Shalev A. FOXO1 competes with carbohydrate response element-binding protein (ChREBP) and inhibits 
thioredoxin-interacting protein (TXNIP) transcription in pancreatic beta cells. J Biol Chem. 2013;288(32):23194-202.

28. Noordeen NA, Meur G, Rutter GA, Leclerc I. Glucose-induced nuclear shuttling of ChREBP is mediated by sorcin and $\mathrm{Ca}(2+)$ ions in pancreatic beta-cells. Diabetes. 2012;61(3):574-85.

29. Ko KP, Kim CS, Ahn Y, Park SJ, Kim YJ, Park JK, et al. Plasma isoflavone concentration is associated with decreased risk of type 2 diabetes in Korean women but not men: results from the Korean genome and epidemiology study. Diabetologia. 2015;58(4):726-35.

30. Álvarez-Cilleros D, López-Oliva E, Goya L, Martín MÁ, Ramos S. Cocoa intake attenuates renal injury in Zucker diabetic fatty rats by improving glucose homeostasis. Food Chem Toxicol. 2019;127:101-9.

31. Pang YL, Hu JW, Liu GL, Lu SY. Comparative medical characteristics of ZDFT2DM rats during the course of development to late stage disease. Animal Model Exp Med. 2018;1(3):203-11.

32. Zheng W, Ma Y, Zhao A, He T, Lyu N, Pan Z. Compositional and functional differences in human gut microbiome with respect to equol production and its association with blood lipid level: a cross-sectional study. Gut Pathog. 2019;10:11-20.

33. Cheong SH, Furuhashi K, Ito K, Nagaoka M, Yonezawa T, Miura Y, Yagasaki K. Antihyperglycemic effect of equol, a daidzein derivative, in cultured L6 myocytes and Ob/Ob mice. Mol Nutr Food Res. 2014;58(2):267-77.

34. Fu Z, Gilbert ER, Liu D. Regulation of insulin synthesis and secretion and pancreatic Beta-cell dysfunction in diabetes. Curr Diabetes Rev. 2013;9(1): 25-53.

35. Jia Q, Yang R, Liu XF, Ma SF. Protective Effects of Genistein on Myocardial Injury in Diabetic Rats. Sichuan da xue xue bao Yi xue ban = Journal of Sichuan University Medical science edition. 2018;49(5):706-11.

36. Liu X, Wang T, Liu X, Cai L, Qi J, Zhang P, et al. Biochanin a protects lipopolysaccharide/D-galactosamine-induced acute liver injury in mice by activating the Nrf2 pathway and inhibiting NLRP3 inflammasome activation. Int Immunopharmacol. 2016;38:324-31.

37. Mousa SA, Gallati C, Simone T, Dier E, Yalcin M, Dyskin E, et al. Dual targeting of the antagonistic pathways mediated by Sirt1 and TXNIP as a putative approach to enhance the efficacy of anti-aging interventions. Aging. 2009;1(4):412-24.

38. Nivet-Antoine V, Cottart CH, Lemarechal H, Vamy M, Margaill I, Beaudeux JL, et al. Trans-resveratrol downregulates Txnip overexpression occurring during liver ischemia-reperfusion. Biochimie. 2010:92(12):1766-71.

39. Ishii S, lizuka K, Miller BC, Uyeda K. Carbohydrate response element binding protein directly promotes lipogenic enzyme gene transcription. Proc Natl Acad Sci U S A. 2004;101(44):15597-602.

40. da Silva XG, Rutter GA, Diraison F, Andreolas C, Leclerc I. ChREBP binding to fatty acid synthase and L-type pyruvate kinase genes is stimulated by glucose in pancreatic beta-cells. J Lipid Res. 2006;47(11):2482-91.

41. Li MV, Chang B, Imamura M, Poungvarin N, Chan L. Glucose-dependent transcriptional regulation by an evolutionarily conserved glucose-sensing module. Diabetes. 2006;55(5):1179-89.

\section{Publisher's Note}

Springer Nature remains neutral with regard to jurisdictional claims in published maps and institutional affiliations.

Ready to submit your research? Choose BMC and benefit from:
- fast, convenient online submission
- thorough peer review by experienced researchers in your field
- rapid publication on acceptance
- support for research data, including large and complex data types
- gold Open Access which fosters wider collaboration and increased citations
- maximum visibility for your research: over 100M website views per year
At BMC, research is always in progress.
Learn more biomedcentral.com/submissions

